\title{
225m Outdoor W-Band Radio-over-Fiber Link Using an Optical SFP+ Module
}

Rommel, Simon; Rodríguez Páez, Juan Sebastián; Chorchos, ukasz; Grakhova, Elizaveta P.; Sultanov, Albert Kh.; Turkiewicz, Jarosaw P.; Vegas Olmos, Juan José; Tafur Monroy, Idelfonso

\section{Published in:}

Proceedings of Optical Fiber Communication Conference and Exhibition 2016

Link to article, DOI:

10.1364/OFC.2016.Th2A.16

Publication date:

2016

Document Version

Peer reviewed version

Link back to DTU Orbit

Citation (APA):

Rommel, S., Rodríguez Páez, J. S., Chorchos, ., Grakhova, E. P., Sultanov, A. K., Turkiewicz, J. P., Vegas Olmos, J. J., \& Tafur Monroy, I. (2016). 225m Outdoor W-Band Radio-over-Fiber Link Using an Optical SFP+ Module. In Proceedings of Optical Fiber Communication Conference and Exhibition 2016 Optical Society of America. https://doi.org/10.1364/OFC.2016.Th2A.16

\section{General rights}

Copyright and moral rights for the publications made accessible in the public portal are retained by the authors and/or other copyright owners and it is a condition of accessing publications that users recognise and abide by the legal requirements associated with these rights.

- Users may download and print one copy of any publication from the public portal for the purpose of private study or research.

- You may not further distribute the material or use it for any profit-making activity or commercial gain

- You may freely distribute the URL identifying the publication in the public portal 


\title{
225m Outdoor W-Band Radio-over-Fiber Link Using an Optical SFP+ Module
}

\author{
Simon Rommel ${ }^{1, *}$, Sebastian Rodriguez ${ }^{1}$, Lukasz Chorchos $^{1,2}$, Elizaveta P. Grakhova ${ }^{1,3}$, \\ Albert Kh. Sultanov ${ }^{3}$, Jarosław P. Turkiewicz ${ }^{2}$, Juan José Vegas Olmos ${ }^{1}$, \\ Idelfonso Tafur Monroy ${ }^{1}$ \\ ${ }^{I}$ Department of Photonics Engineering, Technical University of Denmark, 2800 Kgs. Lyngby, Denmark \\ ${ }^{2}$ Institute of Telecommunications, Warsaw University of Technology, 00-665 Warsaw, Poland \\ ${ }^{3}$ Department of Telecommunication Systems, Ufa State Aviation Technical University, 450000 Ufa, Russia \\ *sirem@fotonik.dtu.dk
}

\begin{abstract}
A W-band radio-over-fiber link based on a commercial SFP+ module is demonstrated, allowing easy integration into existing PON solutions. Without active laser control good RF frequency stability and $225 \mathrm{~m}$ wireless distance are achieved.

OCIS codes: (060.5625) Radio frequency photonics, (060.4510) Optical communications.
\end{abstract}

\section{Introduction}

The increasing use of bandwidth intensive applications—such as high definition video streaming and holographic video conferencing - on consumer devices has created a large demand for high-speed mobile and wireless connections. Recent advances in mobile access technology enable the latter, resulting in the need for high-speed wireless front- and optical backhaul solutions as radio access units need to be adequately connected to the core infrastructure. Additionally these front- and backhaul links must seamlessly tie in with existing distribution networks such as passive optical networks (PONs) [1]. Mm-wave radio-over-fiber (ROF) solutions stand out as a prime candidate as they combine the relatively large available bandwidth in the mm-wave bands with easy optical distribution over significant fiber distances and may coexist with other services on already deployed distribution networks $[1,2]$.

$\mathrm{Mm}$-wave wireless transmissions for front- and backhaul have been demonstrated in a number of configurations $[3,4]$, of which heterodyning of two independent lasers for photonic upconversion is one of the simplest and most promising for integration of ROF links with existing infrastructure. However in oder to truly enable mm-wave backhaul scenarios they need to be based on technology already used in deployed systems, such as SFP+ modules [1].

In this work a W-band hybrid photonic wireless link is demonstrated, based on heterodyne upconversion of the signal of a commercial enhanced small form-factor pluggable (SFP+) - transmitted through $15 \mathrm{~km}$ of fiber-with an independent laser as local oscillator. This is, to the best of our knowledge, the first SFP+-based W-band transmission and at $2.5 \mathrm{Gbit} / \mathrm{s}$ we achieve an outdoor wireless distance of $225 \mathrm{~m}$, limited only by available radio frequency power.

\section{Experimental Setup}

The SFP+ based W-band transmission setup with low complexity in the radio-frequency (RF) domain is depicted in Fig. 1 while Fig. 2 shows the corresponding experimental setup. A commercial SFP+ module is fed with a $2.5 \mathrm{Gbit} / \mathrm{s}$ $2^{15}-1$ bit long pseudo-random bit sequence (PRBS15) non-return-to-zero (NRZ) signal from a pulse pattern generator (PPG) to generate a modulated optical signal which is amplified and transmitted through a combination of $10 \mathrm{~km}$ ITU-T G.652 standard single-mode fiber (SMF) and $5 \mathrm{~km}$ ITU-T G.657.B3 bend insensitive fiber (BIF).

An external cavity laser (ECL) acts as a tunable local oscillator (LO) employed for heterodyne photonic upconversion on a photodiode (PD), with the optical input power $P_{\text {opt }}$ controlled by two variable optical attenuators (VOA). The resulting RF signal is amplified by $8 \mathrm{~dB}$ before being transmitted over distances between $100 \mathrm{~m}$ and $225 \mathrm{~m}$ using a pair of parabolic antennas with a gain of $48 \mathrm{dBi}$ each. At the receiver side a low noise amplifier (LNA) provides $40 \mathrm{~dB}$ gain before the signal is downconverted using a Schottky diode based W-band envelope detector (ED) with a nominal $3 \mathrm{~dB}$ bandwidth of $3 \mathrm{GHz}$. The baseband signal is filtered with a $1.8 \mathrm{GHz}$ Bessel filter to limit noise bandwidth and recorded on a digital storage oscilloscope (DSO).

Using heterodyne photonic upconversion and employing a commercial SFP+ for signal generation the suggested setup minimizes complexity and cost in the optical domain, while maintaining low complexity in the RF domain by using envelope detection. 


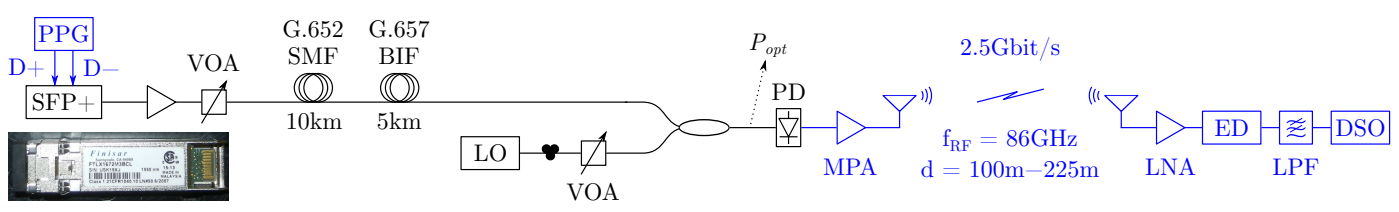

Fig. 1. Experimental setup for radio-over-fiber transmission. SFP+: enhanced small form-factor pluggable, PPG: pulse pattern generator, VOA: variable optical attenuator, LO: local oscillator, PD: photodiode, MPA: medium power amplifier, LNA: low noise amplifier, ED: envelope detector, LPF: Lowpass filter, DSO: digital storage oscilloscope.

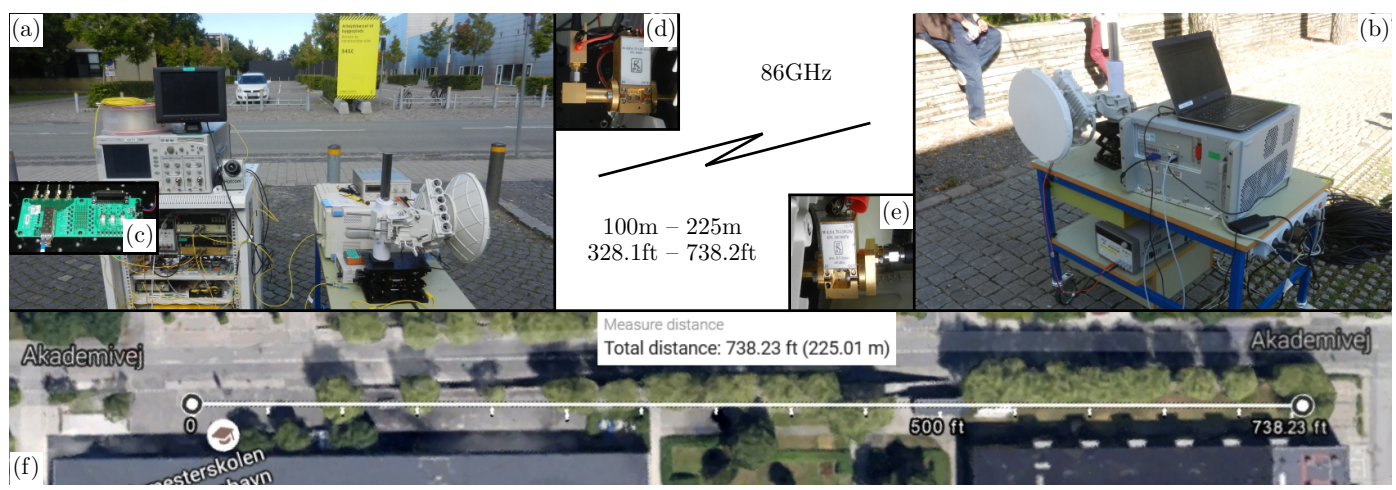

Fig. 2. Outdoor experimental setup: (a) transmitter station, (b) receiver station, (c) evaluation board with SFP+, (d) PD and MPA mounted on transmit antenna, (e) LNA and ED mounted on receive antenna, (f) geographic overview of experiment location and wireless transmission path.

\section{Experimental Results}

Since heterodyne photonic upconversion is performed with two independent lasers and without actively tracking the wavelength of the signal with the LO any wavelength drift of the two lasers may result in a frequency drift of the generated RF signal. Fig. 3 shows the results of an indoor stability measurement over $12 \mathrm{~h}$, finding power stability of the lasers to be within $\pm 0.3 \mathrm{~dB}$ of the set power while a small wavelength drift is observed on both lasers after power on, stabilizing after ca. $2 \mathrm{~h}$ to small variations within $\pm 0.02 \mathrm{~nm}$. The resulting RF carrier frequency is shown in Fig. 3 (d) and found to vary by less than $\pm 0.5 \mathrm{GHz}$ across the whole $12 \mathrm{~h}$ period, despite the uncontrolled drift of laser wavelength observed after power on. This variation is small in comparison to both the bandwidth available in W-band and that of the signal, confirming no active control of the RF generation to be needed.

Hybrid photonic wireless transmission is performed over a total of $15 \mathrm{~km}$ of fiber and wireless distances of $100 \mathrm{~m}$ to $225 \mathrm{~m}$ while monitoring received signal quality. Error free transmission was achieved at all distances with recorded sequences of a total length $>25 \mathrm{Mbit}$, suggesting a BER on the order of $10^{-7}$ or lower. Fig. 4 confirms this by plotting experimentally obtained $Q$ factors over distance alongside resulting BER estimates according to (1), finding an estimated BER of $2.6 \times 10^{-8}$ at $225 \mathrm{~m}$ and even lower error rates for shorter distances.

$$
B E R \approx 3 / 4 \operatorname{erfc}(Q / \sqrt{2})
$$

The observed $Q$ factors suggest a linear relation to distance and thus the system to be limited by received power and additive white gaussian noise. They further confirm and adhere to expectations derived from previous experiments in similar conditions [4].

The presented system setup based on a commercial SFP+ module offers seamless integration of W-band radio access units into existing optical distribution schemes such as PONand WDM point-to-point links based on standard SFP+ pluggables. Despite allowing both lasers to freely drift with no tracking of the signal frequency with the LO the system achieves good frequency stability, allowing reliable transmission of a signal of $5 \mathrm{GHz}$ bandwidth within an allocation of only $6 \mathrm{GHz}$. Allowing for an additional guard band of $1 \mathrm{GHz}$ between channels this suggests an availability of 5 similar channels within the lightly licensed W-band. 

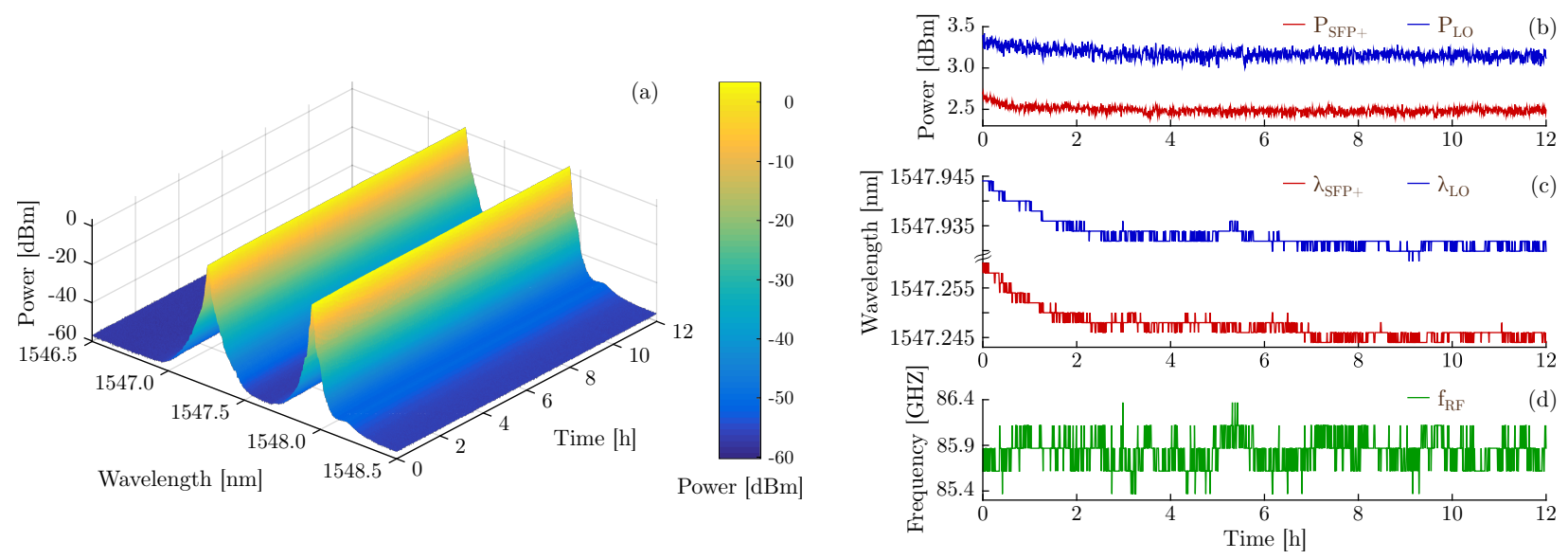

Fig. 3. SFP+ and LO laser power and wavelength and resulting RF carrier frequency stability over $12 \mathrm{~h}$ : (a) recorded spectra, (b) laser powers, (c) laser wavelengths, (d) RF carrier frequency.

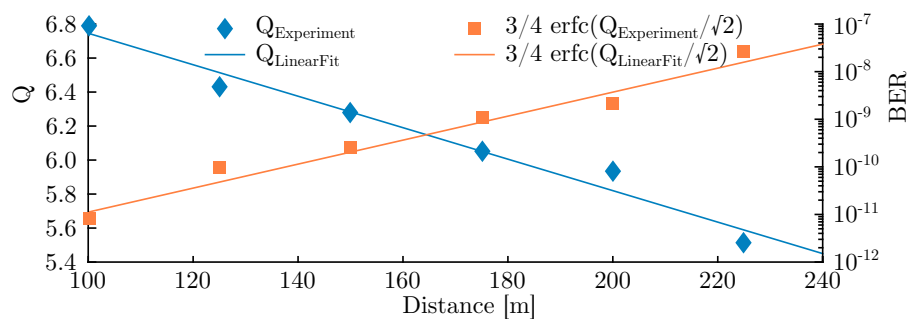

Fig. 4. Observed $Q$ factors and derived BER estimates after $225 \mathrm{~m}$ wireless transmission of a $2.5 \mathrm{Gbit} / \mathrm{s}$ signal on an $86 \mathrm{GHz}$ carrier.

\section{Conclusions}

A RoF link based on a commercial SFP+ module and employing heterodyne photonic upconversion has been demonstrated, including analysis of the frequency stability of the generated RF carrier. The observed stability suggests up to five channels of $5 \mathrm{GHz}$ bandwidth to be available in the W-band and with the achieved transmission distance of $225 \mathrm{~m}$ only limited by available RF transmission power longer distances should be achievable. This highlights the possibility and demonstrates the feasibility of seamlessly extending existing PON structures to enable front- and backhaul to radio access units, based on commercial SFP+ modules.

\section{Acknowledgments}

This work was partly funded by the DFF FTP mmW-SPRAWL and EC IPHOBAC-NG and FiWiN5G projects.

\section{References}

1. M. P. Thakur et al., "Cost-Efficient DWDM-PON / Mm-Wave Wireless Integration using Coherent Radio-overFiber (CRoF)," in Proc. MWP 2015 (IEEE, Paphos, 2015), paper P-19.

2. K.-i. Kitayama, T. Kuri, J. J. Vegas Olmos, and H. Toda, "Fiber-Wireless Networks and Radio-over-Fibre Technique," in Proc. CLEO 2008 (OSA, San Jose, 2008), paper CThR4.

3. A. R. Islam, M. Bakaul, A. Nirmalathas, and G. E. Town, "Simplification of millimeter-wave radio-over-fiber system employing heterodyning of uncorrelated optical carriers and self-homodyning of RF signal at the receiver," Opt. Express 20, 5707-5724 (2012).

4. S. Rommel, L. C. P. Cavalcante, J. J. Vegas Olmos, and I. Tafur Monroy, "Low RF Complexity Photonically Enabled Indoor and Building-to-Building W-Band Wireless Link," in Proc. ACP 2015 (OSA, Hong Kong, 2015), paper AM1B.7. 